\title{
A ZERO DENSITY ESTIMATE FOR DEDEKIND ZETA FUNCTIONS OF PURE EXTENSION FIELDS
}

\author{
By
}

\author{
Koichi KAWADA
}

\section{§1. Introduction}

We first introduce some notation which is utilized throughout this article. Let $k \geq 2$ be a fixed integer, $s=\sigma+i$ be a complex variable with real $\sigma$ and $t, \zeta(s)$ be the Riemann zeta function, $\zeta(s, n)$ be the Dedekind zeta function of the field $\boldsymbol{Q}\left(n^{1 / k}\right)$ where $\boldsymbol{Q}$ denotes the rational number field, and define

$$
\xi(s, n)=\frac{\zeta(s, n)}{\zeta(s)}
$$

By Uchida [10] or van der Waall [11], we see that $\xi(s, n)$ is an entire function for every integer $n$.

The letter $p$ always stands for primes, $\rho(n, p)$ denotes the number of solutions $m(\bmod p)$ to the congruence

$$
m^{k}-n \equiv 0 \quad(\bmod p),
$$

and $\lambda(n, m)$ denotes the completely multiplicative function of $m$ defined by

$$
\lambda(n, p)=\rho(n, p)-1
$$

for all primes $p$.

Let $\mathscr{I}_{k}$ be the set of all integers $n$ such that $x^{k}-n$ is irreducible in $Q[x]$. One may show that $x^{k}-n$ is reducible if, and only if, $n$ is a $p$-th power of an integer for some $p \mid k$, namely,

$$
\mathscr{I}_{k}=\boldsymbol{Z} \backslash\left(\bigcup_{p \mid k} Z^{p}\right)
$$

where $Z$ is the integer ring, and $Z^{p}$ denotes the set of all $p$-th powers of integers. 
In this article we are concerned with the number $\mathscr{N}(n ; \alpha, T)$ of zeros of $\xi(s, n)$ in the region $\sigma \geq \alpha,|t| \leq T$;

$$
\mathscr{N}(n ; \alpha, T)=\#\{s=\sigma+i t ; \quad \xi(s, n)=0, \quad \sigma \geq \alpha, \quad|t| \leq T\} .
$$

By Lemma 3 of [3], the author announced that

$$
\sum_{\substack{n \leq N \\ n \in \mathscr{I}_{k}}} \mathcal{N}(n ; 1-\eta, T) \ll(N T)^{1-\eta}
$$

for $T \leq N^{A}$ with fixed $A>0$, and for some $\eta>0$. (The detailed proof for [3] is contained in the unpublished manuscript [4] which forms a part of author's thesis.) The zero density estimate (2) was prepared for the application to a problem on the representation of numbers as the sum of a prime and a $k$-th power which shall be discussed below.

The main result of this article is a refinement of (2).

THEOREM. Let $k \geq 2$ be an integer, $0<\delta<1 / k, A \geq 1$, and let

$$
\eta=(1000 k A)^{-1} \delta
$$

Then, for $N \geq 1$, and for

$$
N^{(1 / 2)+\delta} \leq H \leq N, \quad 1 \leq T \leq N^{A}, \quad q \in Z, \quad 0<|q| \leq N^{A}
$$

we have

$$
\sum_{\substack{N \leq n \leq N+H \\ n q \in \mathscr{I}_{k}}} \mathcal{N}(n q ; 1-\eta, T) \ll(H T)^{1-\eta}
$$

where the implicit constant is effective, and depends only on $k, A$ and $\delta$.

When $k=2$, the function $\xi(s, n)$ is a Dirichlet $L$ function with a certain real Dirichlet character, and the Theorem is weaker than known results on the zero density for $L$ functions (see Ch. 12 of Montgomery [7], for instance). Our interest is in the case $k \geq 3$.

Our Theorem is closely related to Theorem 1 of Nair and Perelli [8] which states that

$$
\sum_{N \leq n \leq N+H}\left|\sum_{M \leq p \leq 2 M}(\rho(n q, p)-v(n q))\right| \ll H M(\log M)^{-A}
$$


for $M \geq 2$, under the assumptions in the statement of our Theorem, where the implicit constant contained in the Vinogradov symbol is ineffective, and depends on $k, A$ and $\delta$, and where $v(n)$ denotes the number of irreducible factors appearing in the factorization of the polynomial $x^{k}-n$ in $Q[x]$. Note that $v(n)=1$ if, and only if, $n \in \mathscr{I}_{k}$.

The result (5) can be applied to two problems in which our Theorem can work as a substitute for (5), as we see later. Accordingly our Theorem can get rid of ineffective constants in the applications.

Nair and Perelli divided their proof of (5) into two cases according to $M \leq \exp \left(\log N(\log \log N)^{2}\right)$ or not, and the latter case seems more difficult to treat than the former case. For larger $M$, they required to apply the Siegel-Brauer theorem (see Lemmata 3 and 4 of [8]), so that the ineffective constant occurs in (5). For smaller $M$, however, they proved (5) by using a high-moment large sieve inequality (Lemma 1] of [8]), and the proof gives essentially an improvement of Lemma 2] of [3] which was one of the foundations of the proof of (2). By virtue of this improvement (see Lemma 1 below), our Theorem is shown by the same way as the proof of (2), and no ineffective constant occurs in our argument as we are not concerned with too large $M$.

As the proof shows, the exponent $1-\eta$ of $H T$ in the conclusion of our Theorem can be reduced to $1-C \eta$ with larger $C$, but it does not matter, at least, about our applications of the Theorem.

We then observe the applications. We put

$$
S(n, M)=\sum_{m \leq M} \frac{\mu(m)}{\varphi(m)} \lambda(n, m),
$$

where $\mu(m)$ and $\varphi(m)$ denote the Möbius function and Euler's totient function, respectively, and define

$$
S(n)=\prod_{p}\left(1-\frac{\rho(n, p)-1}{p-1}\right)=\prod_{p}\left(1-\frac{\rho(n, p)}{p}\right)\left(1-\frac{1}{p}\right)^{-1},
$$

for $n \in \mathscr{I}_{k}$. The convergence of the infinite product is shown by the prime ideal theorem. And, by the known upper estimate for the residue of $\zeta(s, n)$ at $s=1$ with (31)-(33) of Perelli and Zaccagnini [9], we have $S(n) \gg(\log |n|)^{-k-1}$ for $n \in \mathscr{I}_{k},|n|>1$. On the asymptotic behaviour of the number $R(n)$, say, of the representations of $n$ as the sum of a prime and a $k$-th power, it is expected that

$$
R(n) \sim S(n) \frac{n^{1 / k}}{\log n} \quad\left(\text { as } n \rightarrow \infty, n \in \mathscr{I}_{k}\right) .
$$


In order to show that this expected asymptotic formula is valid for almost every $n$, roughly speaking, it is required to prove that $S(n)$ is satisfactorily approximated by $S(n, M)$ with a suitable parameter $M$ for almost every $n$.

This problem on $\boldsymbol{S}(n)$ was cleared by Miech [6] in 1968 when $k=2$, by using Bombieri's zero density theorem for $L$ functions. And when $k \geq 3$, the estimate (2) works in place of the zero density theorem for $L$ functions, as is announced in [3].

On the other hand, Perelli and Zaccagnini [9] discussed several problems concerning the sum of a prime and a $k$-th power. In particular, applying (5), they proved in [9] that

$$
\boldsymbol{S}(n, M)=\boldsymbol{S}(n)+\boldsymbol{O}\left((\log N)^{-\boldsymbol{A}}\right),
$$

for all but $O\left(H(\log N)^{-A}\right)$ values of $n \in[N, N+H]$ provided $H \geq N^{(1 / 2)+\delta}$, with any fixed $A, \delta>0$, where $M$ is an appropriate parameter, say, $M=N^{1 /(2 k)}$, and where the implicit constants are ineffective and depend on $k, A$ and $\delta$ (see (27), (28), (35), (37) of [9]). This consequence on $S(n)$ is stronger than that contained in [3] with respect to the length of the interval for $n$. Using this, they established in [9] that the foregoing expected asymptotic formula for $R(n)$ is valid for almost every $n \in[N, N+H]$ provided $H \geq N^{1-(1 / k)+\varepsilon}, \varepsilon>0$.

We may give a similar result on $S(n)$ without ineffective constants by applying out Theorem instead of (5).

Corollary 1. Let $1 \leq M \leq H, \eta=\delta /(1000 k)$ and assume the same conditions on $k, \delta, N$ and $H$ as in the Theorem. Then we have

$$
\boldsymbol{S}(n, M)=\boldsymbol{S}(n)+O\left(M^{-\eta / 3}\right),
$$

for all but $O\left(H M^{-\eta / 2}\right)$ values of $n$ in $[N, N+H]$, where the implicit constants are effective, and depend only on $k$ and $\delta$.

Nair and Perelli [8] applied their result (5), that is, Theorem 1 of [8] to another interesting problem on the distribution of the primes represented by an irreducible polynomial, which is concerned with the work of Friedlander and Granville [1]. Theorem 2 of [1] was established under the assumption of the generalized Riemann hypothesis for Dedekind zeta functions, but Nair and Perelli obtained, by applying (5), the same conclusion as Theorem 2 of [1] unconditionally, via their modification of the argument of Maier [5'] (see [8], Theorem 2). In the proof, the result (5) was utilized to show that there is a set $\mathscr{S}_{0}$ satisfying $\# \mathscr{S}_{0}=N+O\left(N(\log N)^{-2}\right)$ and 


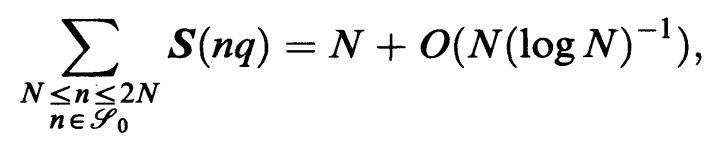

where the implicit constants are again ineffective (see (34) and (36) of [8], and note that, on putting $q=-Q$ and $n=d$, the number $C_{d}=C_{f_{d}}$ in [8] coincides with $S(n q)$ in our notation). And our zero density theorem can work for this part too. For further details on the matter, we refer to [8] as well as [1].

COROllary 2. Let

$$
\mathscr{S}(q)=\left\{n \in[N, N+H] ; \quad \mathscr{N}\left(n q ; 1-\eta, H^{\eta / 2}\right)=0, \quad n q \in \mathscr{I}_{k}\right\} .
$$

Then, under the same assumptions as in the Theorem, we have $\# \mathscr{S}(q)=$ $H+O\left(H^{1-\eta / 2}\right)$, and

$$
\sum_{n \in \mathscr{S}(q)} S(n q)=H+O\left(H^{1-\eta / 3}\right)
$$

where the implicit constants are effective, and depend only on $k, A$ and $\delta$.

We shall prove the Theorem in $\S 4$, and Corollaries in the final section. Besides the notation introduced above, we arrange the following convention. In the sequel, we regard $k, A, \delta$ and $\eta$ as being fixed with the conditions in the statement of the Theorem, or the implicit constants may depend on $k, A$ and $\delta$. We assume that $N$ is sufficiently large in terms of $k, A$ and $\delta$. The letter $\varepsilon$ denotes any sufficiently small positive real number as usual, and is not necesarily the same at each occurrence. In the statements involving $\varepsilon$, the implicit constants may depend on $\varepsilon$ as well.

ACKNOWLEDGEMENT. The author would like to take this opportunity to express his hearty gratitude to Professor Saburô Uchiyama, who retired officially in 1993, and to Dr. Hiroshi Mikawa for their kind, continual support, whilst the author was a graduate student at Tsukuba University.

\section{§2. Fundamental lemma}

The next lemma is the foundation of our argument.

LEMma 1. Let $1 \leq M \leq N^{10 k A}$, and $\left\{a_{m}\right\}$ be any sequence of complex numbers. Then, on (3) and (4), we have 


$$
\sum_{N \leq n \leq N+H}\left|\sum_{M \leq m \leq 2 M} a_{m} \mu(m) \lambda(n q, m)\right| \ll H M^{1-10 \eta} \max _{M \leq m \leq 2 M}\left|a_{m}\right| .
$$

Indeed this is a variant of the result (5) of Nair and Perelli (see [8], §2, case I), and we only remark on the proof briefly.

Let $\mathscr{C}_{m}$ be the set of all the primitive Dirichlet characters $\chi$, modulo $m$, such that $\chi^{k}$ is the principal character. In particular, we arrange that $\mathscr{C}_{1}$ consists of the principal character of modulo 1 . Then one can easily confirm that $\lambda(n q, p)=$ $\sum_{\chi \in \mathscr{C}_{p}} \chi(n q)$, thus

$$
\lambda(n q, m)=\sum_{\chi \in \mathscr{Q}_{m}} \chi(n q)
$$

for all square-free $m$ 's. Putting $b(\chi)=a_{m} \mu(m) \chi(q)$ for $\chi \in \mathscr{C}_{m}$, and $b(\chi)=0$ otherwise, we have, for any integer $l \geq 1$,

$$
\left(\sum_{M \leq m \leq 2 M} \sum_{\chi}|b(x)|^{2 l /(2 l-1)}\right)^{(2 l-1) / 2 l} \ll M^{((2 l-1) / 2 l)+\varepsilon} \max _{M \leq m \leq 2 M}\left|a_{m}\right|,
$$

since $\# \mathscr{C}_{m} \ll m^{\varepsilon}$. Therefore our Lemma 1 is proved by the argument of Nair and Perelli in [8], pp. 5-7 (especially, see [12), (13) and (16) of [8], p. 7).

LEMMA 2. Let $1 \leq M \leq N^{10 k A},\left\{a_{m}\right\}$ be any sequence of complex numbers,

$$
S_{n}(t)=\sum_{M \leq m \leq 2 M} a_{m} \mu(m) \lambda(n q, m) m^{i t},
$$

and, for each $n$, let $\mathscr{T}_{n}$ be a finite set of real numbers in the interval $[-T, T]$ such that $\left|t-t^{\prime}\right| \geq 1$ for all distinct $t, t^{\prime} \in \mathscr{T}_{n}$. Then, on (3) and (4), we have

$$
\sum_{N \leq n \leq N+H} \sum_{t \in \mathscr{T}_{n}}\left|S_{n}(t)\right| \ll H T M^{1-9 \eta} \max _{M \leq m \leq 2 M}\left|a_{m}\right| .
$$

Since Lemma 1.2 of [7] gives

$$
\sum_{t \in \mathscr{T}_{n}}\left|S_{n}(t)\right| \ll \int_{-T-1}^{T+1}\left(\left|S_{n}(t)\right|+\left|\frac{d}{d t} S_{n}(t)\right|\right) d t
$$

Lemma 2 is proved at once by lemma 1 .

\section{§3. Mean value estimate}

This section begins with some basic results concerning $\xi(s, n)$ for $n \in \mathscr{I}_{k}$. We first introduce the function 


$$
\xi_{0}(s, n)=\prod_{p}\left(1-\lambda(n, p) p^{-s}\right)^{-1}=\sum_{m=1}^{\infty} \lambda(n, m) m^{-s},
$$

for $\sigma>1$, and define the numbers $a_{n}(p, f)$ by the Euler product for the Dedekind zeta function $\zeta(s, n)$;

$$
\zeta(s, n)=\prod_{p} \prod_{f=1}^{k}\left(1-p^{-f s}\right)^{-a_{n}(p, f)} \quad(\sigma>1) .
$$

It is known in algebraic number theory that $0 \leq a_{n}(p, f) \leq k$, and that if $p \nmid k n$ and $n \in \mathscr{I}_{k}$ then

$$
a_{n}(p, 1)=\rho(n, p) .
$$

By the last fact and the Euler products for $\zeta(s)$ and $\zeta(s, n)$, we have

$$
\xi_{0}(s, n)=\xi(s, n) F(s, n),
$$

for $\sigma>1, n \in \mathscr{I}_{k}$, where

$$
\begin{aligned}
F(s, n)= & \prod_{p}\left(1-\lambda(n, p) p^{-s}\right)^{-1}\left(1-p^{-s}\right)^{\lambda(n, p)} \cdot \prod_{p \mid k n}\left(1-p^{-s}\right)^{a_{n}(p, 1)-\rho(n, p)} \\
& \cdot \prod_{p} \prod_{f=2}^{k}\left(1-p^{-f s}\right)^{a_{n}(p, f)} .
\end{aligned}
$$

It follows from $0 \leq \rho(n, p) \leq \min (k, p-1)$ and $\rho(n, 2)=1$ that $|\lambda(n, p)| \leq$ $\min (k-1, p-2)$, and that $\left|\lambda(n, p) p^{-s}\right| \leq 1$ when

$$
\sigma=\frac{\log (k-1)}{\log (k+1)}
$$

For our $\eta$, we see $\log (k-1) / \log (k+1)<1-1000 \eta$ by (3). Thus, we may immediately deduce that $F(s, n)$ is regular and

$$
F(s, n) \ll|n|^{\varepsilon},
$$

in the half plane $\sigma \geq 1-100 \eta$. Now the equation (7) provides the analytic continuation of $\xi_{0}(s, n)$ in the half plane $\sigma \geq 1-100 \eta$, which is regular there.

We next show a bound for $\left|\xi_{0}(s, n)\right|$ near the line $\sigma=1$. Let $D_{n}$ be the discriminant of $Q\left(n^{1 / k}\right)$, and let $r_{1}$ and $2 r_{2}$, respectively, be the number of real and complex conjugate fields of $Q\left(n^{1 / k}\right) / Q$, so that $r_{1}+2 r_{2}=k$. By the functional equations of $\zeta(s)$ and $\zeta(s, n)$, we have 


$$
\xi(s, n)=\left(2^{-r_{2}} \pi^{-(k-1) / 2} \sqrt{\left|D_{n}\right|}\right)^{1-2 s}\left(\frac{\Gamma((1-s) / 2)}{\Gamma(s / 2)}\right)^{r_{1}-1}\left(\frac{\Gamma(1-s)}{\Gamma(s)}\right)^{r_{2}} \xi(1-s, n) .
$$

Accordingly, when $\sigma=-\eta$, Stirling's formula gives

$$
\xi(s, n) \ll\left|D_{n}\right|^{(1+2 \eta) / 2}(|t|+1)^{(k-1)(1+2 \eta) / 2},
$$

since $\xi(s, n) \ll 1$ for $\sigma=1+\eta$. As we know $D_{n} \mid D_{n}^{\prime}$, where $D_{n}^{\prime}=(-1)^{k-1} k^{k} n^{k-1}$ is the discriminant of the polynomial $x^{k}-n$, we see $\left|D_{n}\right| \ll|n|^{k-1}$. Then, through a well-known argument based on the Phragmén-Lindelöf theorem, with (7) and (8), we conclude that

$$
\xi_{0}(s, n) \ll(|n|(|t|+1))^{6(k-1) \eta}
$$

for $\sigma \geq 1-10 \eta$ and $n \in \mathscr{I}_{k}$.

The purpose of this section is to obtain the following mean value estimate for $\xi_{0}(s, n)$.

LEMMA 3. For each $n$, let $\mathscr{T}_{n}$ be a finite set of real numbers in the interval $[-T, T]$ such that $\left|t-t^{\prime}\right| \geq 1$ for all distinct $t, t^{\prime} \in \mathscr{T}_{n}$. Then, on (3) and (4), and for $\sigma \geq 1-8 \eta$, we have

$$
\sum_{\substack{N \leq n \leq N+H \\ n q \in \mathscr{F}_{k}}} \sum_{i \in \mathscr{T}_{n}}\left|\xi_{0}(\sigma+i t, n q)\right| \ll H T
$$

Let $M_{0}=N^{9 k A}$, and write

$$
\Xi=\xi_{0}(s+w, n q) \Gamma(w) M_{0}^{w}
$$

for $\sigma \geq 1-8 \eta,|t| \leq T$ and $n q \in \mathscr{I}_{k}$. By shifting the line of integration, and by using (9) with the well-known estimate for the gamma function, we have

$$
\frac{1}{2 \pi i} \int_{2-i \infty}^{2+i \infty} \Xi d w=\xi_{0}(s, n q)+\frac{1}{2 \pi i} \int_{-2 \eta-i \infty}^{-2 \eta+i \infty} \Xi d w=\xi_{0}(s, n q)+O\left(N^{-1}\right) .
$$

On the other hand, by a Mellin transform (see Ivic [2], (A.7)), the above integral turns into

$$
\begin{aligned}
\frac{1}{2 \pi i} \int_{2-i \infty}^{2+i \infty} \Xi d w= & \sum_{m=1}^{\infty} \lambda(n q, m) m^{-s} e^{-m / M_{0}} \\
= & \sum_{m_{2}<\sqrt{M_{1}}} \lambda\left(n q, m_{2}\right)^{2} m_{2}^{-2 s} \sum_{m_{1}<M_{1}} \mu\left(m_{1}\right)^{2} \lambda\left(n q, m_{1}\right) m_{1}^{-s} e^{-m_{1} m_{2}^{2} / M_{0}} \\
& +O\left(N^{-1}\right)
\end{aligned}
$$


since every number $m$ is uniquely written in the form $m=m_{1} m_{2}^{2}$ with $\mu\left(m_{1}\right) \neq 0$, and where $M_{1}$ is the power of 2 satisfying $M_{1} / 2<M_{0}(\log N)^{2} \leq M_{1}$. Consequently, it follows that

$$
\xi_{0}(s, n q) \ll \sum_{m_{2}<\sqrt{M_{1}}} m_{2}^{-2 \sigma+\varepsilon}\left|\sum_{m_{1}<M_{1}} \mu\left(m_{1}\right)^{2} \lambda\left(n q, m_{1}\right) m_{1}^{-s} e^{-m_{1} m_{2}^{2} / M_{0}}\right|+N^{-1}
$$

Since $M_{1}<N^{10 k A}$, Lemma 2 yields

$$
\begin{aligned}
& \sum_{N \leq n \leq N+H} \sum_{t \in \mathscr{T}_{n}}\left|\sum_{M \leq m_{1}<2 M} \mu\left(m_{1}\right)^{2} \lambda\left(n q, m_{1}\right) m_{1}^{-\sigma-i t} e^{-m_{1} m_{2}^{2} / M_{0}}\right| \\
& \ll H T M^{1-9 \eta-\sigma} \ll H T M^{-\eta},
\end{aligned}
$$

for $1 \leq M<M_{1}$, and we have by (10)

$$
\sum_{\substack{N \leq n \leq N+H \\ n q \in \mathscr{T}_{k}}} \sum_{t \in \mathscr{T}_{n}}\left|\xi_{0}(\sigma+i t, n q)\right| \ll H T \sum_{m_{2}<\sqrt{M_{1}}} m_{2}^{-2 \sigma+\eta} \ll H T
$$

as required.

\section{§4. Zero density estimate}

In this section we shall prove the Theorem, Let

$$
h=(\log N)^{2} \text {. }
$$

One can show

$$
\mathscr{N}(n q ; 0, t+1)-\mathscr{N}(n q ; 0, t) \ll \log N
$$

uniformly in $n \in[N, N+H],|t| \leq T$ (see Landau [5], Satz 181). So, for each $n$ satisfying $n q \in \mathscr{I}_{k}$, there is a set $\mathscr{R}_{n}$ having the following three properties:

(i) The set $\mathscr{R}_{n}$ consists of zeros $\rho=\beta+i \gamma$ of $\xi(s, n q)$ with real $\beta$ and $\gamma$ satisfying

$$
\beta \geq 1-\eta, \quad|\gamma| \leq T .
$$

(ii) For all distinct $\rho=\beta+i \gamma$ and $\rho^{\prime}=\beta^{\prime}+i \gamma^{\prime}$ in $\mathscr{R}_{n}$, we have $\left|\gamma-\gamma^{\prime}\right| \geq 3 h$.

(iii) As for the cardinality $\# \mathscr{R}_{n}$, we have

$$
\mathscr{N}(n q ; 1-\eta, T) \ll \# \mathscr{R}_{n} \cdot(\log N)^{3} .
$$

We then put

$$
G_{n}(s)=\sum_{m \leq H T h} \mu(m) \lambda(n q, m) m^{-s}
$$


and write

$$
\Psi=\xi_{0}(\rho+w, n q) G_{n}(\rho+w)(H T)^{w} \Gamma(w),
$$

for $\rho \in \mathscr{R}_{n}, n q \in \mathscr{I}_{k}$, and for $w=u+i v$ with real $u$ and $v$, where $\xi_{0}$ is the function defined in the preceding section. We note that $\Psi$ is regular in the half plane $u \geq$ $1-8 \eta-\beta$ because $\xi_{0}(\rho, n q)=0$ by (7), and that

$$
\xi_{0}(s, n q) G_{n}(s)=1+\sum_{m>H T h} \lambda(n q, m) m^{-s} \sum_{\substack{d \mid m \\ d \leq H T h}} \mu(d),
$$

for $\sigma>1$. Therefore, putting $c=1-8 \eta$, we obtain

$$
\begin{aligned}
\frac{1}{2 \pi i} \int_{c-\beta-i \infty}^{c-\beta+i \infty} \Psi d w & =\frac{1}{2 \pi i} \int_{2-i \infty}^{2+i \infty} \Psi d w \\
& =e^{-(H T)^{-1}}+\sum_{m>H T h} \lambda(n q, m) m^{-\rho} e^{-m(H T)^{-1}} \sum_{\substack{d \mid m \\
d \leq H T h}} \mu(d) \\
& =1+O\left((H T)^{-1}\right) .
\end{aligned}
$$

The integral on the leftmost side also becomes

$$
=\frac{1}{2 \pi i} \int_{c-\beta-i h}^{c-\beta+i h} \Psi d w+O\left(N^{-1}\right),
$$

since $\Gamma(w) \ll e^{-|v|}$. Thus we have

$$
1 \ll \int_{c-\beta-i h}^{c-\beta+i h}|\Psi| d w \ll(H T)^{-7 \eta} \max _{|v| \leq h}\left|\xi_{0}(c+i(\gamma+v), n q) G_{n}(c+i(\gamma+v))\right| .
$$

Hence we may take a real number $t_{\rho}$ such that $\left|t_{\rho}-\gamma\right| \leq h$ and

$$
(H T)^{-7 \eta}\left|\xi_{0}\left(c+i t_{\rho}, n q\right) G_{n}\left(c+i t_{\rho}\right)\right| \gg 1
$$

for each $\rho \in \mathscr{R}_{n}$, which gives

$$
\begin{aligned}
\sum_{\substack{N \leq n \leq N+H \\
n q \in \mathscr{S}_{k}}} \# \mathscr{R}_{n} \ll & (H T)^{-(7 / 2) \eta} \sum_{\substack{N \leq n \leq N+H \\
n q \in \mathcal{S}_{k}}} \sum_{\rho \in \mathscr{P}_{n}}\left|\xi_{0}\left(c+i t_{\rho}, n q\right) G_{n}\left(c+i t_{\rho}\right)\right|^{1 / 2} \\
\ll & (H T)^{-(7 / 2) \eta}\left(\sum_{\substack{N \leq n \leq N+H \\
n \in \in \mathcal{S}_{k}}} \sum_{\rho \in \mathscr{T}_{n}}\left|\xi_{0}\left(c+i t_{\rho}, n q\right)\right|\right)^{1 / 2} \\
& \times\left(\sum_{N \leq n \leq N+H} \sum_{\rho \in \mathscr{P}_{n}}\left|G_{n}\left(c+i t_{\rho}\right)\right|\right)^{1 / 2}
\end{aligned}
$$


The double sums in the parentheses are both $O(H T)$ by Lemmata 3 and 2, as we see that $\left|t_{\rho}-t_{\rho^{\prime}}\right| \geq h$ for all distinct $\rho, \rho^{\prime} \in \mathscr{R}_{n}$, by the property (ii). So we have

$$
\sum_{\substack{N \leq n \leq N+H \\ n q \in \mathcal{G}_{k}}} \# \mathscr{R}_{n} \ll(H T)^{1-(7 / 2) \eta}
$$

which proves our Theorem in view of the property (iii) of the set $\mathscr{R}_{n}$.

\section{§5. Applications}

Lemma 4. Let $n \in \mathscr{I}_{k},|n| \leq H^{4 A}$ and assume that $\mathcal{N}\left(n ; 1-\eta, H^{\eta / 2}\right)=0$ with (3). Then we have

$$
S(n, H)=S(n)+O\left(H^{-\eta / 3}\right) .
$$

As this lemma is proved by fairly familiar arguments, we state the proof somewhat concisely.

Now, set $2 T=H^{\eta / 2}$. By the assumption and (7), the function $\xi_{0}(s, n)$ has no zeros in the region $\sigma \geq 1-\eta,|t| \leq 2 T$, because $F(s, n) \neq 0$ in that region. Then, applying Hadamard's three circle theorem to $\log \xi_{0}(s, n)$, we can show

$$
\xi_{0}(s, n)^{-1} \ll H^{\varepsilon},
$$

for $\sigma \geq 1-\eta / 2,|t| \leq T$ (see the proof of Theorem 1.12 of Ivić [2]). We next define

$$
Z_{n}(s)=\sum_{m=1}^{\infty} \frac{\mu(m) \lambda(n, m)}{\varphi(m) m^{s-1}}
$$

for $\sigma>1$. Handling the Euler product for $Z_{n}(s)$, we have

$$
Z_{n}(s)=\xi_{0}(s, n)^{-1} \prod_{p}\left(1-\frac{\lambda(n, p)}{\left(p^{s}-\lambda(n, p)\right)(p-1)}\right)
$$

which provides the analytic continuation of $Z_{n}(s)$. Particularly, in the region $\sigma \geq$ $1-\eta / 2,|t| \leq T$, it follows that $Z_{n}(s)$ is regular, and that

$$
Z_{n}(s) \ll H^{\varepsilon},
$$

by (12). 
We utilize Perron's formula (see (A.10) of Ivić [2]), and obtain

$$
\begin{aligned}
S(n, H) & =\frac{1}{2 \pi i} \int_{\eta / 7-i T}^{\eta / 7+i T} Z_{n}(s+1) \frac{H^{s}}{s} d s+O\left(H^{\eta / 7} T^{-1}\right) \\
& =S(n)+\frac{1}{2 \pi i}\left(I_{1}+I_{2}+I_{3}\right)+O\left(H^{-\eta / 3}\right)
\end{aligned}
$$

since $S(n)=Z_{n}(1)$, where

$$
\begin{aligned}
& I_{1}=\int_{\eta / 7-i T}^{-\eta / 2-i T} Z_{n}(s+1) \frac{H^{s}}{s} d s, \quad I_{2}=\int_{-\eta / 2-i T}^{-\eta / 2+i T} Z_{n}(s+1) \frac{H^{s}}{s} d s, \\
& I_{3}=\int_{-\eta / 2+i T}^{\eta / 7+i T} Z_{n}(s+1) \frac{H^{s}}{s} d s .
\end{aligned}
$$

The bound (13) yields $I_{j} \ll H^{\eta / 7+\varepsilon} T^{-1} \ll H^{-\eta / 3}$ for $j=1,3$, and $I_{2} \ll$ $H^{-\eta / 2+\varepsilon} \log T \ll H^{-\eta / 3}$, thus the lemma follows from (14).

Finally, we prove the Corollaries. Our Theorem gives

$$
\sum_{\substack{N \leq n \leq N+H \\ n q \in \mathscr{F}_{k}}} \mathcal{N}\left(n q ; 1-\eta, H^{\eta / 2}\right) \ll H^{1-\eta / 2}
$$

on (3) and (4), and we may readily observe by (1) that the number of $n$ 's in $[N, N+H]$ with $n q \notin \mathscr{I}_{k}$ is $O(H / \sqrt{N})$. Therefore, as to the cardinality of the set $\mathscr{S}(q)$ which is defined in the statement of Corollary 2, we obtain

$$
\# \mathscr{S}(q)=H+O\left(H^{1-\eta / 2}\right) .
$$

Then, using Lemma 4, (15) and the estimate $S(n q, H) \ll H^{\varepsilon}$ which holds even if $n \notin \mathscr{S}(q)$, we have

$$
\begin{aligned}
\sum_{n \in \mathscr{S}(q)} S(n q) & =\sum_{n \in \mathscr{S}(q)} S(n q, H)+O\left(H^{1-\eta / 3}\right) \\
& =\sum_{N \leq n \leq N+H} S(n q, H)+O\left(H^{1-\eta / 3}\right) .
\end{aligned}
$$

By (6), we see

$$
S(n q, H)=1+\sum_{2 \leq m \leq H} \frac{\mu(m)}{\varphi(m)} \sum_{\chi \in \mathscr{B}_{m}} \chi(n q)
$$


so that

$$
\begin{aligned}
\sum_{N \leq n \leq N+H} S(n q, H) & =H+O(1)+\sum_{2 \leq m \leq H} \frac{\mu(m)}{\varphi(m)} \sum_{\chi \in \mathscr{C}_{m}} \chi(q) \sum_{N \leq n \leq N+H} \chi(n) \\
& =H+O\left(H^{(1 / 2)+\varepsilon}\right)
\end{aligned}
$$

by the Pólya-Vinogradov inequality. Now we complete the proof of Corollary 2 by (16) and the last formula.

We turn to Corollary 1 . We then put $q=A=1$. Lemma 4 states that (11) holds for every $n \in \mathscr{S}(1)$, while lemma 1 gives

$$
\sum_{N \leq n \leq N+H}|S(n, H)-S(n, M)| \ll H M^{-9 \eta}
$$

for $1 \leq M \leq H$. From these things with (15), Corollary 1 may be deduced easily.

\section{References}

[ 1 ] Friedlander, J. and Granville, A., Limitations to equi-distribution of primes IV, Proc. Roy. Soc. London Ser. A 435 (1991), 197-204.

[2] Ivić, A., "The Riemann Zeta-Function." New York, Wiley, 1985.

[3] Kawada, K., On the asymptotic formula for the number of representations of numbers as the sum of a prime and a $k$-th power, Proc. Japan Acad. 69 Ser. A (1993), 283-286.

[4] Kawada, K., On the sum of a prime and a $k$-th power, Mathematical Research Note, Inst. of Math., Univ. of Tsukuba, No. 92-008 (unpubished).

[5] Landau, E., "Einführung in die Elementare und Analytische Theorie der Algebraischen Zahlen und der Ideale." Chelsea, New York, 1949.

[ 5'] Maier, H., Primes in short interrals, Michigan Math. J. 32 (1985), 221-225.

[6] Miech, R. J., On the equation $n=p+x^{2}$, Trans. Amer. Math. Soc. 130 (1968), 494-512.

[7] Montgomery, H. L., "Topics in Multiplicative Number Theory." Springer-Verlag, BerlinHeidelberg-New York, 1971.

[8] Nair, M. and Perelli, A., On the prime ideal theorem and irregularities in the distribution of primes, Duke Math. J. 77 (1995), 1-20.

[ 9 ] Perelli, A. and Zaccagnini, A., On the sum of a prime and a $k$-th power, Izvestija Acad. Nauk. Ser. Mat. 59 (1995), 185-200.

[10] Uchida, K., On Artin $L$-functions, Tôhoku Math. J. 27 (1975), 75-81.

[11] van der Waall, R. W., On a conjecture of Dedekind on zeta functions, Indag. Math. 37 (1975), 83-86.

Department of Mathematics, Faculty of Education, Iwate University, Morioka, 020-8550 Japan.

E-mail: kawada@iwate-u.ac.jp 